\title{
Some Local Properties of Soft Semi-Open Sets
}

\author{
Bin Chen \\ School of Mathematical Sciences, University of Jinan, Jinan 250022, China \\ Correspondence should be addressed to Bin Chen; jnchenbin@yahoo.com.cn
}

Received 4 March 2013; Accepted 31 March 2013

Academic Editor: Shurong Sun

Copyright (C) 2013 Bin Chen. This is an open access article distributed under the Creative Commons Attribution License, which permits unrestricted use, distribution, and reproduction in any medium, provided the original work is properly cited.

We introduce some local properties by soft semi-open sets. For example, soft semi-neighborhoods of the soft point, soft semi-firstcountable spaces and soft semi-pu-continuous at the soft point are given. Furthermore, we define soft semi-connectedness and prove that a soft topological space is soft semiconnected if and only if both soft semi-open and soft semi-closed sets are only $\emptyset$ and $\widetilde{X}$.

\section{Introduction}

Soft set theory [1] was firstly introduced by Molodtsov in 1999 as a general mathematical tool for dealing with uncertain, fuzzy, not clearly defined objects. He has shown several applications of this theory in solving many practical problems in economics, engineering, social science, medical science, and so forth, in [1].

In the recent years, papers about soft sets theory and their applications in various fields have been writing increasingly [2-6]. Shabir and Naz [7] introduced the notion of soft topological spaces which are defined over an initial universe with a fixed set of parameters. The authors introduced the definitions of soft open sets, soft closed sets, soft interior, soft closure, and soft separation axioms. And the authors got some important results for soft separation axioms. The results are valuable for research in this field. Tanay and Kandemir [8] introduced the definition of fuzzy soft topology over a subset of the initial universe set while Jun et al. [9] studied the ideal theory in BCK/BCI-Algebras based on soft sets. Along the line of Shabir and Naz, Chen introduced the notations of soft semi-open sets in soft topological spaces in [10].

In the present study, we introduce some local properties by soft semi-open sets. For example, soft semi-neighborhoods of the soft point, soft semi-first-countable spaces, and soft semi-pu-continuous at the soft point are given. And some of their properties are studied.

\section{Preliminaries}

Let $U$ be an initial universe set and $E_{U}$ be a collection of all possible parameters with respect to $U$, where parameters are the characteristics or properties of objects in $U$. We will call $E_{U}$ the universe set of parameters with respect to $U$.

Definition 1 (see [1]). A pair $(F, A)$ is called a soft set over $U$ if $A \subset E_{U}$ and $F: A \rightarrow P(U)$, where $P(U)$ is the set of all subsets of $U$.

Definition 2 (see [1]). Let $U$ be an initial universe set and $E_{U}$ be a universe set of parameters. Let $(F, A)$ and $(G, B)$ be soft sets over a common universe set $U$ and $A, B \subset E$. Then $(F, A)$ is a subset of $(G, B)$, denoted by $(F, A) \widetilde{C}(G, B)$, if: (i) $A \subset B$; (ii) for all $e \in A, F(e) \subset G(e)$.

$(F, A)$ equals $(G, B)$, denoted by $(F, A)=(G, B)$, if $(F, A) \widetilde{\subset}(G, B)$ and $(G, B) \widetilde{\subset}(F, A)$.

Definition 3 (see [1]). A soft set $(F, A)$ over $U$ is called a null soft set, denoted by $\emptyset$, if $e \in A, F(e)=\emptyset$.

Definition 4 (see [1]). A soft set $(F, A)$ over $U$ is called an absolute soft set, denoted by $\widetilde{A}$, if $e \in A, F(e)=U$. 
Definition 5 (see [1]). The union of two soft sets $(F, A)$ and $(G, B)$ over a common universe $U$ is the soft set $(H, C)$, where $C=A \cup B$, and for all $e \in C$,

$$
H(e)= \begin{cases}F(e), & \text { if } e \in A-B, \\ G(e), & e \in B-A, \\ F(e) \cup G(e), & e \in B \cap A .\end{cases}
$$

We write $(F, A) \cup(G, B)=(H, C)$.

Definition 6 (see [1]). The intersection of two soft sets of $(F, A)$ and $(G, B)$ over a common universe $U$ is the soft set $(H, C)$, where $C=A \cap B$, and for all $e \in C, H(e)=F(e) \cap G(e)$. We write $(F, A) \cap(G, B)=(H, C)$.

Now we recall some definitions and results defined and discussed in $[6,7,10]$. Henceforth, let $X$ be an initial universe set and $E$ be the fixed nonempty set of parameter with respect to $X$ unless otherwise specified.

Definition 7. For a soft set $(F, A)$ over $U$, the relative complement of $(F, A)$ is denoted by $(F, A)^{\prime}$ and is defined by $(F, A)^{\prime}=\left(F^{\prime}, A\right)$, where $F^{\prime}: A \rightarrow P(U)$ is a mapping given by $F^{\prime}(e)=U-F(e)$ for all $e \in A$.

Definition 8. Let $\tau$ be the collection of soft sets over $X$, then $\tau$ is called a soft topology on $X$ if $\tau$ satisfies the following axioms.

(1) $\emptyset, \widetilde{X}$ belong to $\tau$.

(2) The union of any number of soft sets in $\tau$ belongs to $\tau$.

(3) The intersection of any two soft sets in $\tau$ belongs to $\tau$. $X$.

The triplet $(X, \tau, E)$ is called a soft topological space over

Definition 9. Let $(X, \tau, E)$ be a soft space over $X$, then the members of $\tau$ are said to be soft open sets in $X$.

Definition 10. Let $(X, \tau, E)$ be a soft space over $X$. A soft set $(F, E)$ over $X$ is said to be a soft closed set in $X$, if its relative complement $(F, E)^{\prime}$ belongs to $\tau$.

Proposition 11. Let $(X, \tau, E)$ be a soft space over $X$. Then one has the following.

(1) $\emptyset, \widetilde{X}$ are soft closed sets over $X$.

(2) The intersection of any number of soft closed sets is a soft closed set over $X$.

(3) The union of any two soft closed sets is a soft closed set over $X$.

Definition 12. Let $(X, \tau, E)$ be a soft topological space and $(A, E)$ be a soft set over $X$.

(1) The soft interior of $(A, E)$ is the soft set $\operatorname{Int}(A, E)=$ $\bigcup\{(O, E):(O, E)$ is soft open and $(O, E) \widetilde{\subset}(A, E)\}$.
(2) The soft closure of $(A, E)$ is the soft set $\mathrm{Cl}(A, E)=$ $\bigcap\{(F, E):(F, E)$ is soft closed and $(A, E) \widetilde{C}(F, E)\}$.

By property 2 for soft open sets, $\operatorname{Int}(A, E)$ is soft open. It is the largest soft open set contained in $(A, E)$.

By property 2 for soft closed sets, $\mathrm{Cl}(A, E)$ is soft closed. It is the smallest soft closed set containing $(A, E)$.

Proposition 13. Let $(X, \tau, E)$ be a soft topological space and let $(F, E)$ and $(G, E)$ be a soft set over $X$. Then

(1) $\operatorname{Int}(\operatorname{Int}(F, E))=\operatorname{Int}(F, E)$,

(2) $(F, E) \widetilde{\subset}(G, E)$ implies $\operatorname{Int}(F, E) \widetilde{\subset} \operatorname{Int}(G, E)$,

(3) $\mathrm{Cl}(\mathrm{Cl}(F, E))=\mathrm{Cl}(F, E)$,

(4) $(F, E) \widetilde{C}(G, E)$ implies $\mathrm{Cl}(F, E) \widetilde{C} \mathrm{Cl}(G, E)$.

Definition 14. A soft set $(A, E)$ in a soft topological space $(X, \tau, E)$ will be termed soft semi-open (written S.S.O) if and only if there exists a soft open set $(O, E)$ such that $(O, E) \widetilde{\subset}(A, E) \widetilde{C} \mathrm{Cl}(O, E)$.

Definition 15. A soft set $(B, E)$ in a soft topological space $(X, \tau, E)$ will be termed soft semi-closed (written S.S.C) if its relative complement is soft semi-open, for example, there exists a soft closed set $(F, E)$ such that $\operatorname{Int}(F, E) \widetilde{\subseteq}(B, E) \widetilde{C}$ $(F, E)$.

Definition 16. Let $(X, \tau, E)$ be a soft topological space and $(A, E)$ be a soft set over $X$.

(1) The soft semi-interior of $(A, E)$ is the soft set $\operatorname{Int}_{S}(A, E)=\bigcup\{(O, E):(O, E)$ is soft semi-open and $(O, E) \widetilde{c}(A, E)\}$.

(2) The soft semi-closure of $(A, E)$ is the soft set $\mathrm{Cl}_{S}(A, E)=\bigcap\{(F, E):(F, E)$ is soft semi-closed and $(A, E) \widetilde{C}(F, E)\}$.

\section{Soft Semi-Neighborhoods}

Definition 17. A soft set $(F, E)$ in a soft topological space $(X, \tau, E)$ is said to be a soft semi-neighborhood of the soft point $e_{F}$ if there is a soft semi-open set $(B, E)$ s.t. $e_{F} \widetilde{\epsilon}$ $(B, E) \widetilde{\widetilde{c}}(F, E)$.

The semi-neighborhood system of a soft point $e_{F}$ which is denoted by $\mathcal{U}_{e_{F}}$ is the set of all its semi-neighborhoods.

Proposition 18. The semi-neighborhood system $\mathcal{U}_{e_{F}}$ at a soft point $e_{F}$ in the soft topological space $(X, \tau, E)$ has the following results.

(a) If $(F, E) \in \mathscr{U}_{e_{F}}$, then one has $e_{F} \tilde{\epsilon}(F, E)$.

(b) If $(F, E) \in \mathcal{U}_{e_{F}}$ and $(F, E) \widetilde{\subseteq}(B, E)$, then one has $(B, E) \in \mathcal{U}_{e_{F}}$

(c) If $(F, E) \in \mathcal{U}_{e_{F}}$, then there exists a soft $\operatorname{set}(B, E) \in \mathcal{U}_{e_{F}}$ s.t. $(F, E) \in \mathscr{U}_{e^{\prime}}$ for each $e^{\prime} \tilde{\epsilon}(B, E)$.

Proof. (a) If $(F, E) \in \mathcal{U}_{e_{F}}$, then we have a soft semi-open set $(B, E)$ s.t. $e_{F} \tilde{\epsilon}(B, E) \widetilde{\subseteq}(F, E)$. So we have $e_{F} \tilde{\epsilon}(F, E)$. 
(b) If $(F, E) \in \mathscr{U}_{e_{F}}$ and $(F, E) \widetilde{\subseteq}(B, E)$. Because $(F, E) \epsilon$ $\mathcal{U}_{e_{F}}$, there exists a soft semi-open set $(H, E)$ s.t. $e_{F} \tilde{\epsilon}$ $(H, E) \widetilde{\subseteq}(F, E)$. So we have $e_{F} \widetilde{\epsilon}(H, E) \widetilde{\subseteq}(F, E) \widetilde{\subseteq}(B, E)$ and we have $(B, E) \in \mathscr{U}_{e_{F}}$.

(c) If $(F, E) \in \mathcal{U}_{e_{F}}$, then there is a soft semi-open set $(H, E)$ s.t. $e_{F} \tilde{\epsilon}(H, E) \widetilde{\subseteq}(F, E)$. Let $(B, E)=(H, E)$ and for each $e^{\prime} \tilde{\epsilon}(B, E), e^{\prime} \tilde{\epsilon}(B, E) \widetilde{\subseteq}(H, E) \widetilde{\subseteq}(F, E)$. This shows $(F, E) \in \mathscr{U}_{e^{\prime}}$.

Definition 19. Let $(X, \tau, E)$ be a soft topological space and $\mathscr{U}_{e_{E}}$ be a soft semi-neighborhood of a soft point $e_{F}$. If for every soft semi-neighborhood $(F, E)$ of $e_{F}$, there is a $(H, E) \in \mathscr{V}_{e_{F}} \subseteq$ $\mathcal{U}_{e_{F}}$ such that $e_{F} \tilde{\epsilon}(H, E) \widetilde{\subseteq}(F, E)$, then $\mathscr{V}_{e_{F}}$ is said to be a soft semi-neighborhoods base of $\mathscr{U}_{e_{F}}$ at $e_{F}$.

Proposition 20. Let $(X, \tau, E)$ be a soft topological space and $\mathcal{U}_{e_{F}}$ be a soft semi-neighborhood of a soft point $e_{F} . \mathscr{V}_{e_{F}}$ is the soft semi-neighborhoods base of $\mathcal{U}_{e_{F}}$ at $e_{F}$. Then one has the following.

(a) If $(F, E) \in \mathscr{V}_{e_{F}}$, then we have $e_{F} \tilde{\epsilon}(F, E)$.

(b) If $(F, E) \in \mathscr{V}_{e_{F}}$ and $(F, E) \widetilde{\simeq}(B, E)$, then one has $(B, E) \in \mathscr{V}_{e_{F}}$.

(c) If $(F, E) \in \mathscr{V}_{e_{F}}$, then there exists a soft set $(B, E) \in \mathscr{V}_{e_{F}}$ s.t. $(F, E) \in \mathscr{V}_{e^{\prime}}$ for each $e^{\prime} \tilde{\epsilon}(B, E)$.

Proof. These properties are easily verified by referring to the corresponding properties of soft seminbds in Proposition 18.

Definition 21. Let $(X, \tau, E)$ be a soft topological space and $e_{F}$ be a soft point in $(X, \tau, E)$. If $e_{F}$ has a countable soft semineighborhoods base, then we say that $(X, \tau, E)$ is soft semifirst-countable at $e_{F}$. If each soft point in $(X, \tau, E)$ is soft semifirst-countable, then we say that $(X, \tau, E)$ is a soft semi-firstcountable space.

Proposition 22. Let $(X, \tau, E)$ be a soft topological space and $e_{F}$ be a soft point in $(X, \tau, E)$. Then $(X, \tau, E)$ is soft semifirst-countable at $e_{F}$ if and only if there is a countable soft semi-neighborhoods base $\left\{\left(F_{n}, E\right), n \in N\right\}$ at $e_{F}$ such that $\left(F_{n+1}, E\right) \widetilde{\simeq}\left(F_{n}, E\right)$ for each $n \in N$.

Proof. $\Leftarrow$ Obvious.

$\Rightarrow$ Let $\left\{\left(U_{n}, E\right), n \in N\right\}$ be a countable soft semineighborhoods base at $e_{F}$. For each $n \in N$, put $\left(F_{n}, E\right)=$ $\bigcap_{i=1}^{n}\left(U_{i}, E\right)$. Then it is easy to see that $\left\{\left(F_{n}, E\right), n \in N\right\}$ is a soft semi-neighborhoods base at $e_{F}$ and $\left(F_{n+1}, E\right) \widetilde{\subseteq}\left(F_{n}, E\right)$ for each $n \in N$.

Definition 23. Let $(X, \tau, E)$ be a soft topological space, $(B, E)$ be a soft set, and $e_{F}$ be a soft point in $(X, \tau, E)$. Then $e_{F}$ is said to be a soft semi-interior point of $(B, E)$ if there is a soft semiopen set $(H, E)$ satisfies $e_{F} \tilde{\epsilon}(H, E) \widetilde{\subseteq}(B, E)$.
Proposition 24. Let $e_{F}$ be a soft point in $(X, \tau, E)$ and $(B, E)$ be a soft semi-open set. Then one has the following results.

(a) Each soft point $e_{F} \tilde{\epsilon}(B, E)$ is a soft semi-interior point.

(b) For each $e \in E$, one can define $(B,\{e\})$ as follows:

$$
B\left(e^{\prime}\right)= \begin{cases}B(e), & \text { if } e^{\prime}=e, \\ \emptyset, & \text { if } e^{\prime} \neq e .\end{cases}
$$

Then $(B,\{e\})$ is a soft semi-interior point of $(B, E)$ and $(B,\{e\})=\cup e_{F}$ for each soft semi-interior point $e_{F}$ of $(B, E)$.

(c) $\cup_{e \in E}(B,\{e\})=(B, E)$.

Proof. (a), (c) are obvious by definitions.

(b) For $(B, E)$ is a soft semi-open set, the soft semi-interior point $(B,\{e\})$ is the largest soft semi-interior point of $(B, E)$ by $e \in E$ and we have $(B,\{e\})=\cup e_{F}$ for every soft semi-interior point $e_{F}$ of $(B, E)$.

Proposition 25. Let $(X, \tau, E)$ be a soft topological space, $(B, E)$ be a soft set. Then $\operatorname{Int}_{S}(B, E)=\bigcup_{e \in E}\left\{e_{F}: e_{F}\right.$ is any soft semiinterior point of $(B, E)\}$.

Proof. By the definition of soft semi-interior and Proposition 24.

Definition 26. A soft set $(F, E)$ in a soft topological space $(X, \tau, E)$ is called a soft semi-neighborhood (briefly: snbd) of the soft set $(G, E)$ if there is a soft semi-open set $(B, E)$ s.t. $(G, E) \widetilde{\simeq}(B, E) \widetilde{\simeq}(F, E)$.

Proposition 27. Let $(X, \tau, E)$ be a soft topological space, $(B, E)$ be a soft set. Then $(B, E)$ is soft semi-open if and only iffor each soft set $(F, E)$ contained in $(B, E),(B, E)$ is a soft semi-nbd of $(F, E)$.

Proof. $\Rightarrow$ obvious

$\Leftarrow$ Because $(B, E) \widetilde{\subseteq}(B, E)$, then we have a soft semi-open set $(H, E)$ s.t. $(B, E) \widetilde{\subseteq}(H, E) \widetilde{\subseteq}(B, E)$. So we have $(B, E)=$ $(H, E)$ and $(B, E)$ is soft semi-open.

Definition 28 (see [6]). Let the sequence $\left\{\left(F_{n}, E\right), n \in N\right\}$ be a soft sequence in a soft topological space $(X, \tau, E)$. Then $\left\{\left(F_{n}, E\right), n \in N\right\}$ is eventually contained in a soft set $(B, E)$ if and only if there is an integer $m$ such that, if $n \geq m$, then $\left(F_{n}, E\right) \widetilde{\simeq}(B, E)$. The sequence is frequently contained in $(B, E)$ if and only if for each integer $m$, there is an integer $n$ such that $n \geq m$ and $\left(F_{n}, E\right) \widetilde{\subseteq}(B, E)$.

Definition 29. Let the sequence $\left\{\left(F_{n}, E\right), n \in N\right\}$ be a soft sequence in a soft topological space $(X, \tau, E)$, then one says $\left\{\left(F_{n}, E\right), n \in N\right\}$ semiconverges to a soft point $e_{F}$ if it is eventually contained in each semi-nbd of $e_{F}$. And the soft point $e_{F}$ is said to be a semicluster soft point of $\left\{\left(F_{n}, E\right), n \in\right.$ $N\}$ if the sequence is frequently contained in every semi-nbd of $e_{F}$. 
Proposition 30. Let $(X, \tau, E)$ be soft semi-first-countable, then one has the following.

(a) $(B, E)$ is soft semi-open $\Leftrightarrow$ for every soft sequence $\left\{\left(F_{n}, E\right), n \in N\right\}$ which semiconverges to $e_{F}$ in $(B, E)$ is eventually contained in $(B, E)$.

(b) If $e_{F}$ is a semicluster soft point of the soft sequence $\left\{\left(F_{n}, E\right), n \in N\right\}$, then one has a subsequence of $\left\{\left(F_{n}, E\right), n \in N\right\}$ which semi-converges to $e_{F}$.

Proof. (a) $\Rightarrow$ Because $(B, E)$ is soft semi-open, $(B, E)$ is a seminbd of $e_{F}$, and $\left\{\left(F_{n}, E\right), n \in N\right\}$ semi-converges to $e_{F}$. Then we have $\left\{\left(F_{n}, E\right), n \in N\right\}$ is eventually contained in $(B, E)$.

$\Leftarrow$ For each $e_{F}$ contained in $(B, E)$, let $\left\{\left(F_{n}, E\right), n \in N\right\}$ be the semi-nbd systems such that $\left(F_{n+1}, E\right) \widetilde{\subseteq}\left(F_{n}, E\right)$ for each $n \in N$ by Proposition 22. Then $\left\{\left(F_{n}, E\right), n \in N\right\}$ is eventally contained in each semi-nbd of $e_{F}$ that is, $\left\{\left(F_{n}, E\right), n \in N\right\}$ semi-converges to $e_{F}$. So we have an integer $m$ such that, if $n \geq m,\left(F_{n}, E\right) \widetilde{\subseteq}(B, E)$. Then $(B, E)$ is a semi-nbd of $e_{F}$, and by Proposition $27,(B, E)$ is soft semi-open.

(b) Let $\left\{\left(F_{n}, E\right)\right\}, n \in N$ be the seminbds such that $\left(F_{n+1}, E\right) \widetilde{\subseteq}\left(F_{n}, E\right)$ for each $n \in N$ by Proposition 22. For every nonnegative integer $i$, find $f(i)$ satisfies $f(i) \geq i$ and $\left(F_{f(i)}, E\right) \widetilde{\subseteq}\left(F_{i}, E\right)$. Then $\left\{\left(F_{f(i)}, E\right), i \in N\right\}$ is a subsequence of the sequence $\left\{\left(F_{n}, E\right), n \in N\right\}$. Obviously this subsequence semiconverges to $e_{F}$.

\section{Soft Semi- $p u$-Continuous Functions}

In this part, we define the soft semi- $p u$-continuous functions induced by two mapping $u: X \rightarrow Y$ and $p: E_{1} \rightarrow E_{2}$ on soft topological spaces $\left(X, \tau_{1}, E_{1}\right),\left(Y, \tau_{2}, E_{2}\right)$ and study some properties on them. We use $S S(X)_{E_{1}}$ and $S S(Y)_{E_{2}}$ denote all soft sets on $\left(X, E_{1}\right)$ and $\left(Y, E_{2}\right) . S S S(X)_{E_{1}}$ and $S S S(Y)_{E_{2}}$ denote all semi-open soft sets on soft topological spaces $\left(X, \tau_{1}, E_{1}\right)$ and $\left(Y, \tau_{2}, E_{2}\right)$.

Definition 31. Let $\left(X, \tau_{1}, E_{1}\right)$ and $\left(Y, \tau_{2}, E_{2}\right)$ be two soft topological spaces. Let $u: X \rightarrow Y$ and $p: E_{1} \rightarrow E_{2}$ be mappings. Let $f_{p u}: S S(X)_{E_{1}} \rightarrow S S(Y)_{E_{2}}$ be a function and $e_{F}$ be a soft point in $\left(X, \tau_{1}, E_{1}\right)$.

(a) $f_{p u}$ is soft semi-pu-continuous at $e_{F}$ if for any $\left(B, E_{2}\right) \in \mathcal{U}_{f_{p u}\left(e_{F}\right)}^{\tau_{2}}$, there is a $\left(F, E_{1}\right) \in \mathcal{U}_{e_{F}}^{\tau_{1}}$ s.t. $f_{p u}((F$, $\left.\left.E_{1}\right)\right) \widetilde{\subseteq}\left(B, E_{2}\right)$.

(b) $f_{p u}$ is soft semi-pu-continuous from $\left(X, \tau_{1}, E_{1}\right)$ to $\left(Y, \tau_{2}, E_{2}\right)$ if $f_{p u}$ is soft semi-pu-continuous for every soft point of $\left(X, \tau_{1}, E_{1}\right)$.

Proposition 32. Let $\left(X, \tau_{1}, E_{1}\right)$ and $\left(Y, \tau_{2}, E_{2}\right)$ be two soft topological spaces. Let $u: X \rightarrow Y$ and $p: E_{1} \rightarrow E_{2}$ be mappings. Let $f_{p u}: S S(X)_{E_{1}} \rightarrow S S(Y)_{E_{2}}$ be a function and $e_{F}$ be a soft point in $\left(X, \tau_{1}, E_{1}\right)$. Then the following statements are equal.

(a) $f_{p u}$ is soft semi-pu-continuous at $e_{F}$.

(b) For any $\left(B, E_{2}\right) \in \mathcal{U}_{f_{p u}\left(e_{F}\right)}^{\tau_{2}}$, there is a $\left(F, E_{1}\right) \in \mathcal{U}_{e_{F}}^{\tau_{1}}$ satisfies $\left(F, E_{1}\right) \widetilde{\simeq} f_{p u}^{-1}\left(B, E_{2}\right)$.

(c) For any $\left(B, E_{2}\right) \in \mathcal{U}_{f_{p u}\left(e_{F}\right)}^{\tau_{2}}, f_{p u}^{-1}\left(B, E_{2}\right) \in \mathcal{U}_{e_{F}}^{\tau_{1}}$.

Proof. From Definition 31, this is obvious.

Proposition 33. Let $\left(X, \tau_{1}, E_{1}\right)$ and $\left(Y, \tau_{2}, E_{2}\right)$ be two soft topological spaces. Let $u: X \rightarrow Y$ and $p: E_{1} \rightarrow E_{2}$ be mappings. $f_{p u}: S S(X)_{E_{1}} \rightarrow S S(Y)_{E_{2}}$ be a function. Then the following statements are equal.

(a) $f_{p u}$ is soft semi-pu-continuous.

(b) If $\left(B, E_{2}\right) \in S S S(Y)_{E_{2}}$, one has $f_{p u}^{-1}\left(\left(B, E_{2}\right)\right) \in S S S(X)_{E_{1}}$.

(c) For each soft semi-closed set $\left(F, E_{2}\right) \in\left(Y, \tau_{2}, E_{2}\right)$, one has $f_{p u}^{-1}\left(\left(F, E_{2}\right)\right)$ is soft semi-closed in $\left(X, \tau_{1}, E_{1}\right)$.

Proof. (a) $\Rightarrow$ (b) Let $\left(B, E_{2}\right) \in S S S(Y)_{E_{2}}$ and $e_{F} \tilde{\epsilon} f_{p u}^{-1}((B$, $\left.\left.E_{2}\right)\right)$. Next, we will prove that $f_{p u}^{-1}\left(\left(B, E_{2}\right)\right) \in \mathcal{U}_{e_{F}}^{\tau_{1}}$. Because $f_{p u}\left(e_{F}\right) \tilde{\epsilon}\left(B, E_{2}\right)$ and $\left(B, E_{2}\right) \in S S S(Y)_{E_{2}}$, we have $\left(B, E_{2}\right) \in$ $\mathcal{U}_{f_{p u}\left(e_{F}\right)}^{\tau_{2}}$. Because $f_{p u}$ is soft semi-pu-continuous at $e_{F}$, there is a $\left(F, E_{1}\right) \in \mathcal{U}_{e_{F}}^{\tau_{1}}$ s.t. $f_{p u}\left(\left(F, E_{1}\right)\right) \widetilde{\subseteq}\left(B, E_{2}\right)$. So we have $e_{F} \tilde{\epsilon}\left(F, E_{1}\right) \widetilde{\subseteq} f_{p u}^{-1}\left(\left(B, E_{2}\right)\right)$ and $f_{p u}^{-1}\left(\left(B, E_{2}\right)\right) \in \mathcal{U}_{e_{F}}^{\tau_{1}}$. By Proposition 27, $f_{p u}^{-1}\left(\left(B, E_{2}\right)\right) \in S S S(X)_{E_{1}}$.

(b) $\Rightarrow$ (a) Let $e_{F}$ be a soft point, $\left(B, E_{2}\right) \in \mathcal{U}_{f_{p u}\left(e_{F}\right)}^{\tau_{2}}$. Then we can get a soft semi-open set $\left(F, E_{2}\right) \in S S S(Y)_{E_{2}}$ s.t. $f_{p u}\left(e_{F}\right) \tilde{\epsilon}\left(F, E_{2}\right) \widetilde{\subseteq}\left(B, E_{2}\right)$. By (b) $f_{p u}^{-1}\left(\left(F, E_{2}\right)\right) \in S S S(X)_{E_{1}}$ and $e_{F} \tilde{\epsilon} f_{p u}^{-1}\left(\left(F, E_{2}\right)\right) \widetilde{\subseteq} f_{p u}^{-1}\left(\left(B, E_{2}\right)\right)$. And we have $f_{p u}^{-1}((B$, $\left.\left.E_{2}\right)\right) \in \mathcal{U}_{e_{F}}^{\tau_{1}}$. So, we prove that $f_{p u}$ is soft semi-pu-continuous at each soft point.

(b) $\Rightarrow$ (c) Let $\left(F, E_{2}\right)$ be soft semi-closed in $\left(Y, \tau_{2}, E_{2}\right)$. Then $\left(F, E_{2}\right)^{\prime} \in S S S(Y)_{E_{2}}$ and by (b), $f_{p u}^{-1}\left(\left(F, E_{2}\right)^{\prime}\right) \in S S S(X)_{E_{1}}$. Because $f_{p u}^{-1}\left(\left(F, E_{2}\right)^{\prime}\right)=\left(f_{p u}^{-1}\left(\left(F, E_{2}\right)\right)\right)^{\prime}$, we prove $f_{p u}^{-1}((F$, $\left.\left.E_{2}\right)\right)$ is soft semi-closed in $\left(X, \tau_{1}, E_{1}\right)$.

(c) $\Rightarrow$ (b) Using the same method in (b) $\Rightarrow$ (c), we can get the result.

Proposition 34. Let $\left(X, \tau_{1}, E_{1}\right)$ and $\left(Y, \tau_{2}, E_{2}\right)$ be two soft topological spaces. Let $u: X \rightarrow Y$ and $p: E_{1} \rightarrow E_{2}$ be mappings. $f_{p u}: S S(X)_{E_{1}} \rightarrow S S(Y)_{E_{2}}$ be a function. If $(X, \tau, E)$ is soft semi-first-countable, then the following statements are equal.

(a) $f_{p u}$ is soft semi-pu-continuous.

(b) If $\left(B, E_{1}\right) \in\left(X, \tau_{1}, E_{1}\right)$, the inverse image of any seminbd of $f_{p u}\left(\left(B, E_{1}\right)\right)$ is a semi-nbd of $\left(B, E_{1}\right)$.

(c) If $\left(B, E_{1}\right) \in\left(X, \tau_{1}, E_{1}\right)$ and for every semi-nbd $\left(F, E_{2}\right)$ of $f_{p u}\left(\left(B, E_{1}\right)\right)$, there exists a semi-nbd $\left(G, E_{1}\right)$ of $\left(B, E_{1}\right)$ satisfies $f_{p u}\left(\left(G, E_{1}\right)\right) \widetilde{\subseteq}\left(F, E_{2}\right)$. 
(d) Let the soft sequence $\left\{\left(F_{n}, E_{1}\right), n \in N\right\}$ semi-converges to $e_{F}$, then one has $\left\{f_{p u}\left(F_{n}, E_{1}\right), n \in N\right\}$ semi-converges to $f_{p u}\left(e_{F}\right)$.

Proof. (a) $\Rightarrow$ (b) Let $f_{p u}$ be soft semi-pu-continuous. If $\left(F, E_{2}\right)$ is a semi-nbd of $f_{p u}\left(\left(B, E_{1}\right)\right)$, then $\left(F, E_{2}\right)$ contains a soft semi-open nbd $\left(H, E_{2}\right)$ of $f_{p u}\left(\left(B, E_{1}\right)\right)$. Because $f_{p u}((B$, $\left.\left.E_{1}\right)\right) \widetilde{\subseteq}\left(H, E_{2}\right) \widetilde{\subseteq}\left(F, E_{2}\right)$, we have $f_{p u}^{-1}\left(f_{p u}\left(\left(B, E_{1}\right)\right)\right) \widetilde{\subseteq} f_{p u}^{-1}((H$, $\left.\left.E_{2}\right)\right) \widetilde{\subseteq} f_{p u}^{-1}\left(\left(F, E_{2}\right)\right)$. Since $\left(B, E_{1}\right) \widetilde{\subseteq} f_{p u}^{-1}\left(f_{p u}\left(\left(B, E_{1}\right)\right)\right)$ and $f_{p u}^{-1}\left(\left(H, E_{2}\right)\right)$ is soft semi-open. So, we have $f_{p u}^{-1}\left(\left(F, E_{2}\right)\right)$ is a semi-nbd of $\left(B, E_{1}\right)$.

(b) $\Rightarrow$ (c) Let $\left(B, E_{1}\right) \in\left(X, \tau_{1}, E_{1}\right)$ be a soft set and $\left(F, E_{2}\right)$ be any semi-nbd of $f_{p u}\left(\left(B, E_{1}\right)\right)$. Then by $(\mathrm{b}), f_{p u}^{-1}\left(\left(F, E_{2}\right)\right)$ is a semi-nbd of $\left(B, E_{1}\right)$. So there is a soft semi-open set $\left(G, E_{1}\right) \in$ $\left(X, \tau_{1}, E_{1}\right)$ s.t. $\left(B, E_{1}\right) \widetilde{\subseteq}\left(G, E_{1}\right) \widetilde{\subseteq} f_{p u}^{-1}\left(\left(F, E_{2}\right)\right)$. So we have $f_{p u}\left(\left(G, E_{1}\right)\right) \widetilde{\subseteq}\left(F, E_{2}\right)$.

$(\mathrm{c}) \Rightarrow(\mathrm{d})$ Let $\left(B, E_{2}\right)$ be a semi-nbd of $\left\{f_{p u}\left(e_{F}\right)\right\}$, then there exists a semi-nbd $\left(H, E_{1}\right)$ of $e_{F}$ s.t. $f_{p u}\left(\left(H, E_{1}\right)\right) \widetilde{\subseteq}\left(B, E_{2}\right)$. Since the sequence of soft sets $\left\{\left(F_{n}, E_{1}\right), n \in N\right\}$ semi-converges to $e_{F}$, there is an $m$ such that $n \geq m$, for the semi-nbd $\left(H, E_{1}\right)$ of $e_{F},\left(F_{n}, E_{1}\right) \widetilde{\subseteq}\left(H, E_{1}\right)$. So we have $f_{p u}\left(\left(F_{n}, E_{1}\right)\right) \widetilde{\subseteq} f_{p u}\left(\left(H, E_{1}\right)\right) \widetilde{\subseteq}\left(B, E_{2}\right)$. Thus, $\left\{f_{p u}\left(F_{n}, E_{1}\right), n \in N\right\}$ semi-converges to $f_{p u}\left(e_{F}\right)$.

$(\mathrm{d}) \Rightarrow$ (a) Let $\left(H, E_{2}\right)$ be any semi-open soft set over $\left(Y, \tau_{2}, E_{2}\right)$. We show $f_{p u}^{-1}\left(\left(H, E_{2}\right)\right)$ is semi-open soft set over $\left(X, \tau_{1}, E_{1}\right)$. Let $e_{F}$ be any soft point of $f_{p u}^{-1}\left(\left(H, E_{2}\right)\right)$. Because $(X, \tau, E)$ is semi-soft first-countable, then there exists a countable soft semi-neighborhoods base $\left\{\left(F_{n}, E\right), n \in N\right\}$ at $e_{F}$ such that $\left(F_{n+1}, E\right) \widetilde{\simeq}\left(F_{n}, E\right)$ for each $n \in N$. Because $\left\{\left(F_{n}, E\right), n \in N\right\}$ is decreasing, there exists an $m$ such that for $n \geq m,\left(F_{n}, E\right) \widetilde{\subseteq} f_{p u}^{-1}\left(\left(H, E_{2}\right)\right)$. We know each $\left(F_{n}, E\right)$ is soft semi-neighborhood of $e_{F}$, so $f_{p u}^{-1}\left(\left(H, E_{2}\right)\right)$ is a soft semineighborhood of $e_{F}$ by Proposition 18 (b). This complete the proof.

Proposition 35. Let $\left(X, \tau_{1}, E_{1}\right)$ and $\left(Y, \tau_{2}, E_{2}\right)$ be two soft topological spaces. Let $u: X \rightarrow Y$ and $p: E_{1} \rightarrow E_{2}$ be mappings. $f_{p u}: S S(X)_{E_{1}} \rightarrow S S(Y)_{E_{2}}$ be a function. Then the following statements are equal.

(a) $f_{p u}$ is soft semi-pu-continuous.

(b) If $\left(A, E_{1}\right) \in\left(X, \tau_{1}, E_{1}\right)$, one has $f_{p u}\left(\mathrm{Cl}_{S}\left(A, E_{1}\right)\right) \widetilde{c}$ $\mathrm{Cl}_{S} f_{p u}\left(\left(A, E_{1}\right)\right)$.

(c) If $\left(B, E_{2}\right) \in\left(Y, \tau_{2}, E_{2}\right)$, one has $\mathrm{Cl}_{S} f_{p u}^{-1}\left(\left(B, E_{2}\right)\right) \tilde{c}$ $f_{p u}^{-1}\left(\mathrm{Cl}_{S}\left(B, E_{2}\right)\right)$.

(d) If $\left(B, E_{2}\right) \in\left(Y, \tau_{2}, E_{2}\right)$, one has $f_{p u}^{-1}\left(\operatorname{Int}_{S}\left(B, E_{2}\right)\right) \widetilde{c}$ Int $_{S} f_{p u}^{-1}\left(\left(B, E_{2}\right)\right)$.

Proof. (a) $\Rightarrow$ (b) For $f_{p u}$ is soft semi-pu-continuous, then by Proposition 33 (c), $f_{p u}^{-1}\left(\mathrm{Cl}_{S} f_{p u}\left(\left(A, E_{1}\right)\right)\right)$ is soft semi-closed containing $\left(A, E_{1}\right)$, and thus $\mathrm{Cl}_{S}\left(A, E_{1}\right) \widetilde{\subset} f_{p u}^{-1}\left(\mathrm{Cl}_{S} f_{p u}((A\right.$, $\left.\left.\left.E_{1}\right)\right)\right)$, which gives $f_{p u}\left(\mathrm{Cl}_{S}\left(A, E_{1}\right)\right) \widetilde{\subset} f_{p u} f_{p u}^{-1}\left(\mathrm{Cl}_{S} f_{p u}((A\right.$, $\left.\left.\left.E_{1}\right)\right)\right) \widetilde{\subset} \mathrm{Cl}_{S} f_{p u}\left(\left(A, E_{1}\right)\right)$.
To prove that (b) $\Rightarrow$ (c), we apply (b) to $\left(A, E_{1}\right)=$ $f_{p u}^{-1}\left(\left(B, E_{2}\right)\right)$ and we obtain the inclusion $f_{p u}\left(\mathrm{Cl}_{S} f_{p u}^{-1}((B\right.$, $\left.\left.\left.E_{2}\right)\right)\right) \widetilde{\subset} \mathrm{Cl}_{S} f_{p u}\left(f_{p u}^{-1}\left(\left(B, E_{2}\right)\right)\right) \widetilde{\subset} \mathrm{Cl}_{S}\left(B, E_{2}\right)$, which gives $\mathrm{Cl}_{S} f_{p u}^{-1}\left(\left(B, E_{2}\right)\right) \tilde{\subset} f_{p u}^{-1}\left(\mathrm{Cl}_{S}\left(B, E_{2}\right)\right)$.

To prove that $(\mathrm{c}) \Rightarrow(\mathrm{d})$, we apply $(\mathrm{c})$ to $\left(B, E_{2}\right)^{\prime}$ and we obtain the inclusion $\mathrm{Cl}_{S} f_{p u}^{-1}\left(\left(B, E_{2}\right)^{\prime}\right) \widetilde{\subset} f_{p u}^{-1}\left(\mathrm{Cl}_{S}\left(B, E_{2}\right)^{\prime}\right)$, which gives $f_{p u}^{-1}\left(\operatorname{Int}_{S}\left(B, E_{2}\right)\right)=f_{p u}^{-1}\left(\left(\mathrm{Cl}_{S}\left(B, E_{2}\right)^{\prime}\right)^{\prime}\right)=\left(f_{p u}^{-1}\left(\mathrm{Cl}_{S}(B\right.\right.$, $\left.\left.\left.E_{2}\right)^{\prime}\right)\right)^{\prime} \widetilde{C}\left(\mathrm{Cl}_{S} f_{p u}^{-1}\left(\left(B, E_{2}\right)^{\prime}\right)\right)^{\prime}=\left(\mathrm{Cl}_{S}\left(\left(f_{p u}^{-1}\left(\left(B, E_{2}\right)\right)\right)^{\prime}\right)\right)^{\prime}=$ $\operatorname{Int}_{S} f_{p u}^{-1}\left(\left(B, E_{2}\right)\right)$.

To complete $(\mathrm{d}) \Rightarrow$ (a), for every $\left(B, E_{2}\right) \in S S S(Y)_{E_{2}}$ we have $\left(B, E_{2}\right)=\operatorname{Int}_{S}\left(B, E_{2}\right)$, and it follows from (d) that $f_{p u}^{-1}\left(\left(B, E_{2}\right)\right) \widetilde{\subset} \operatorname{Int}_{S} f_{p u}^{-1}\left(\left(B, E_{2}\right)\right)$. Thus, we have $f_{p u}^{-1}\left(\left(B, E_{2}\right)\right)=$ Int $_{S} f_{p u}^{-1}\left(\left(B, E_{2}\right)\right)$, that is, $f_{p u}^{-1}\left(\left(B, E_{2}\right)\right)$ is soft semi-open in $\left(X, \tau_{1}, E_{1}\right)$. Then $f_{p u}$ is soft semi-pu-continuous.

\section{Soft Semi-Connectedness}

Definition 36. Let $(X, \tau, E)$ be a soft topological space. A soft semiseparation on $\widetilde{X}$ is a pair $(A, E)$ and $(B, E)$ of nonnull soft semi-open sets s.t. $\widetilde{X}=(A, E) \bigcup(B, E),(A, E) \bigcap(B, E)=\emptyset$.

Definition 37. A soft topological space $(X, \tau, E)$ is called soft semi-connected space if there is no soft semiseparations on $\widetilde{X}$. And if $(X, \tau, E)$ has such soft semiseparations, then $(X, \tau, E)$ is called soft semi-disconnected space.

Theorem 38. Let $(X, \tau, E)$ be a soft topological space. $(A, E)$ and $(B, E)$ are semiseparations on $\widetilde{X}$. If $(F, E)$ is a soft semiconnected subspace of $(X, \tau, E)$, then one has $(F, E) \widetilde{\widetilde{\subseteq}}(A, E)$ or $(F, E) \widetilde{\simeq}(B, E)$.

Proof. Because $(A, E)$ and $(B, E)$ are soft semi-open sets, then we have $(F, E) \cap(A, E)$ and $(F, E) \cap(B, E)$ are also soft semi-open sets. Hence $(F, E) \cap(A, E)$ and $(F, E) \cap(B, E)$ are semiseparations of $(F, E)$. And this is a contradiction. So, one of $(F, E) \cap(A, E)$ and $(F, E) \cap(B, E)$ is $\emptyset$ and thus $(F, E) \widetilde{\subseteq}(A, E)$ or $(F, E) \widetilde{\simeq}(B, E)$.

Theorem 39. Let $(X, \tau, E)$ be a soft topological space and $(F, E)$ is a soft semi-connected subspace of $(X, \tau, E)$.

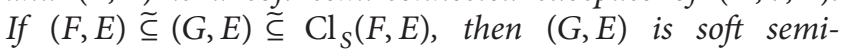
connected.

Proof. Suppose $(G, E)$ is not soft semi-connected, then there exist nonnull soft semi-open sets $(A, E)$ and $(B, E)$ which form a soft semiseparation of $(G, E)$. Then by Theorem 38, we have $(F, E) \widetilde{\subseteq}(A, E)$ or $(F, E) \widetilde{\subseteq}(B, E)$. Suppose $(F, E) \widetilde{\simeq}(A, E)$, then we have $(G, E) \widetilde{\widetilde{c}} \mathrm{Cl}_{S}(F, E) \widetilde{\subseteq} \mathrm{Cl}_{S}(A, E)$. So $(G, E) \cap(B, E) \widetilde{\simeq} \mathrm{Cl}_{S}(A, E) \cap(B, E)=(A, E) \cap(B, E)=\emptyset$. Hence $(B, E)=(B, E) \cap(G, E)=\emptyset$, a contradiction. Thus, we have $(G, E)$ is soft semi-connected.

Theorem 40. A soft topological space $(X, \tau, E)$ is soft semiconnected if and only if the both soft semi-open and soft semiclosed soft sets are only $\emptyset$ and $\widetilde{X}$. 
Proof. $\Rightarrow$ Let the soft topological space $(X, \tau, E)$ be soft semiconnected. If $(A, E)$ is both soft semi-open and soft semiclosed in $(X, \tau, E)$ which is different from $\emptyset$ and $\widetilde{X}$. So $(A, E)^{\prime}$ is a soft semi-open set in $(X, \tau, E)$ which is different from $\emptyset$ and $\widetilde{X}$. Then $(A, E)$ and $(A, E)^{\prime}$ is a soft semiseparation of $(X, \tau, E)$. This is a contradiction. So the both soft semi-open and soft semi-closed soft sets are only $\emptyset$ and $\widetilde{X}$.

$\Leftarrow$ Let $(A, E)$ and $(B, E)$ be a soft semiseparation of $(X, \tau, E)$. Let $(A, E) \neq \widetilde{X}$ and by Definition $36(A, E)=(B, E)^{\prime}$. This proves that $(A, E)$ is both soft semi-open and soft semiclosed in $(X, \tau, E)$ which is different from $\emptyset$ and $\widetilde{X}$. This is a contradiction. So $(X, \tau, E)$ is soft semi-connected.

Corollary 41. A soft topological space $\left(X, \tau_{1}, E_{1}\right)$ is soft semidisconnected if and only if there exists a nonnull proper soft subset which is both soft semi-open and semi-closed.

Proof. By Theorem 40.

Theorem 42. Let $\left(X, \tau_{1}, E_{1}\right)$ and $\left(Y, \tau_{2}, E_{2}\right)$ be two soft topological spaces. $f_{p u}: S S(X)_{E_{1}} \rightarrow S S(Y)_{E_{2}}$ be a soft semi-pucontinuous function. If $\left(X, \tau_{1}, E_{1}\right)$ is soft semi-connected, then $\left(Y, \tau_{2}, E_{2}\right)$ is soft semi-connected.

Proof. Suppose that $\left(Y, \tau_{2}, E_{2}\right)$ is not soft semi-connected. Then there is a soft semiseparation $\left(A, E_{2}\right)$ and $\left(B, E_{2}\right)$ of $\left(Y, \tau_{2}, E_{2}\right)$. So we have $\widetilde{X}=f_{p u}^{-1}\left(\left(A, E_{2}\right) \cup\left(B, E_{2}\right)\right)=$ $f_{p u}^{-1}\left(A, E_{2}\right) \bigcup f_{p u}^{-1}\left(B, E_{2}\right)$ and $f_{p u}^{-1}\left(A, E_{2}\right) \cap f_{p u}^{-1}\left(B, E_{2}\right)=$ $f_{p u}^{-1}(\tilde{Y})=\emptyset_{X}$. Obviously, $f_{p u}^{-1}\left(A, E_{2}\right)$ and $f_{p u}^{-1}\left(B, E_{2}\right)$ are different from $\emptyset_{X}$. So $f_{p u}^{-1}\left(A, E_{2}\right)$ and $f_{p u}^{-1}\left(B, E_{2}\right)$ are a soft semiseparation of $\left(X, \tau_{1}, E_{1}\right)$. This forms a contradiction. Thus, $\left(Y, \tau_{2}, E_{2}\right)$ is soft semi-connected.

\section{Concluding Remarks}

In this paper, we introduce the concept of soft semi-neighborhoods of the soft point, soft semi-pu-continuous at the soft point and soft semi-connectedness. And some of their properties are studied. In the study, soft semi-first-countable space is also given. And in the following papers, soft Frechét space, soft sequential space, soft set-Frechét space and the tightness of a soft point and their connection with soft semifirst-countable space need further study. We hope that the results in this paper will be useful in practical life and nature society.

\section{Acknowledgments}

This work described here is supported by the Grants from the National Natural Science Foundation of China (NSFC no. 10971186, 11061004, 71140004, 61070241, 11226265), the Natural Scientific Foundation of Shandong Province (ZR2010AM019, ZR2010FM035, ZR2011AQ015, ZR2010AQ012, and 2012BSB01159). This work is also supported by the international cooperation in training projects for outstanding young teachers of higher institutions of Shandong Province. The author is grateful to the anonymous referees for careful checking of the details and for helpful comments that improved this paper.

\section{References}

[1] D. Molodtsov, "Soft set theory-first results," Computers \& Mathematics with Applications, vol. 37, no. 4-5, pp. 19-31, 1999.

[2] P. K. Maji, R. Biswas, and A. R. Roy, "Fuzzy soft sets," Journal of Fuzzy Mathematics, vol. 9, no. 3, pp. 589-602, 2001.

[3] B. Ahmad and A. Kharal, "On fuzzy soft sets," Advances in Fuzzy Systems, vol. 2009, Article ID 586507, 6 pages, 2009.

[4] X. Zhou, Q. Li, and L. Guo, "On generalized interval-valued fuzzy soft sets," Journal of Applied Mathematics, vol. 2012, Article ID 479783, 18 pages, 2012.

[5] S. Alkhazaleh, A. R. Salleh, and N. Hassan, "Possibility fuzzy soft set," Advances in Decision Sciences, vol. 2011, Article ID 479756, 18 pages, 2011.

[6] İ. Zorlutuna, M. Akdag, W. K. Min, and S. Atmaca, "Remarks on soft topological spaces," Annals of Fuzzy Mathematics and Informatics, vol. 3, no. 2, pp. 171-185, 2012.

[7] M. Shabir and M. Naz, "On soft topological spaces," Computers \& Mathematics with Applications, vol. 61, no. 7, pp. 1786-1799, 2011.

[8] B. Tanay and M. B. Kandemir, "Topological structure of fuzzy soft sets," Computers \& Mathematics with Applications, vol. 61, no. 10, pp. 2952-2957, 2011.

[9] Y. B. Jun, K. J. Lee, and M. S. Kang, "Ideal theory in BCK/BCIalgebras based on soft sets and $N$-structures," Discrete Dynamics in Nature and Society, vol. 2012, Article ID 910450, 13 pages, 2012.

[10] B. Chen, "Soft semi-open sets and related properties in soft topological spaces," Applied Mathematics \& Information Sciences, vol. 7, no. 1, pp. 287-294, 2013. 


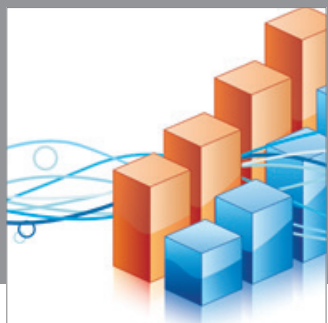

Advances in

Operations Research

mansans

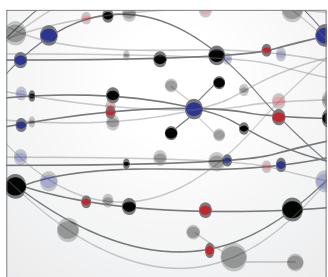

The Scientific World Journal
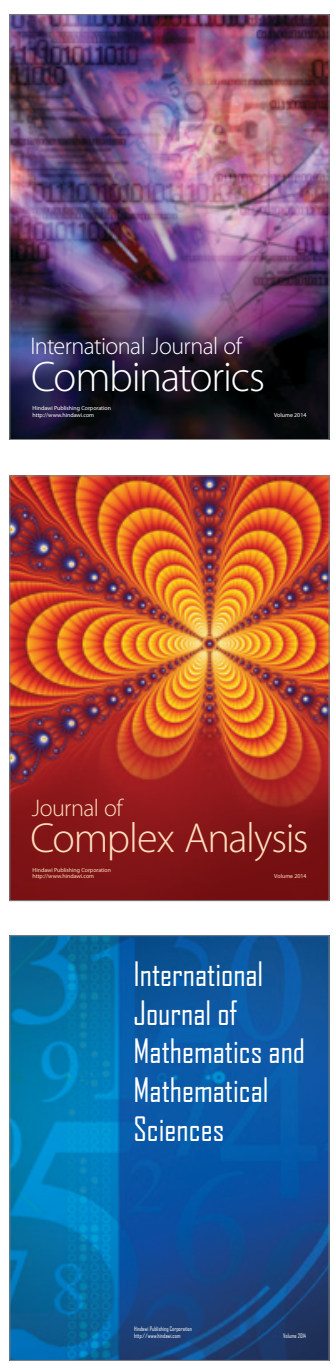
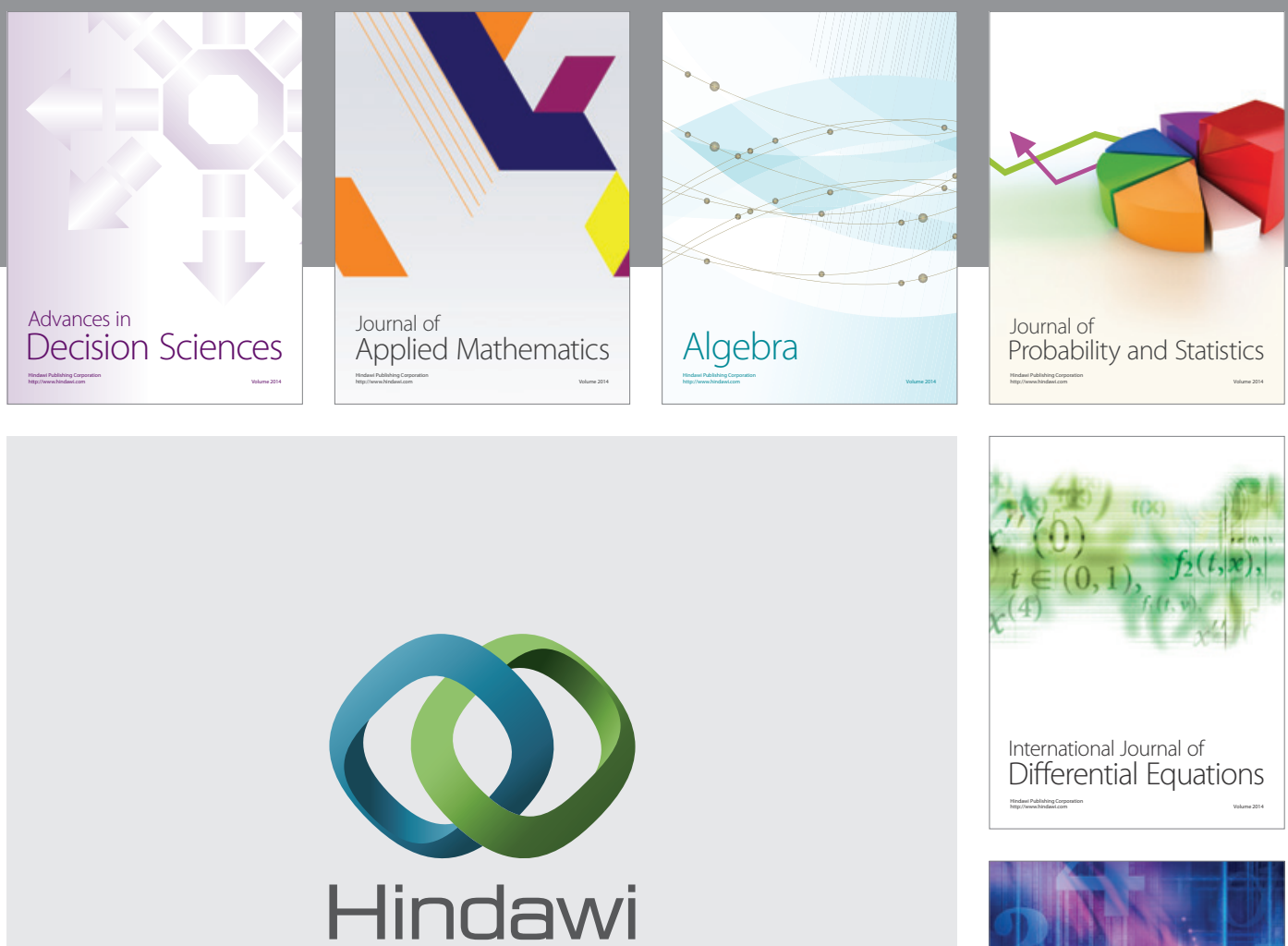

Submit your manuscripts at http://www.hindawi.com
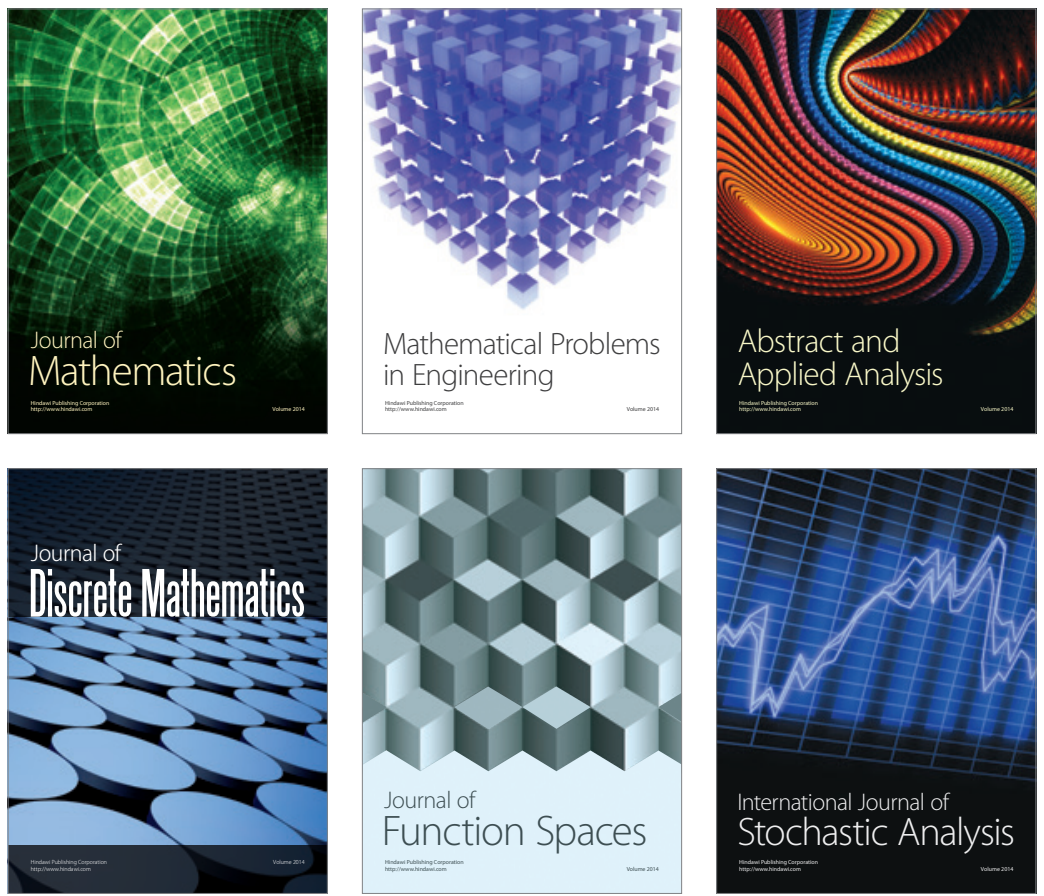

Journal of

Function Spaces

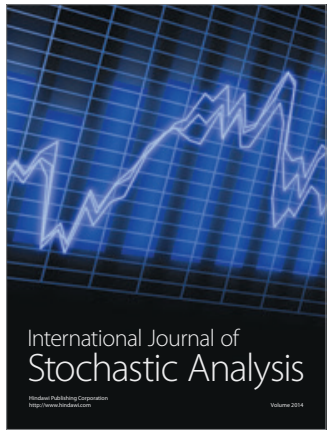

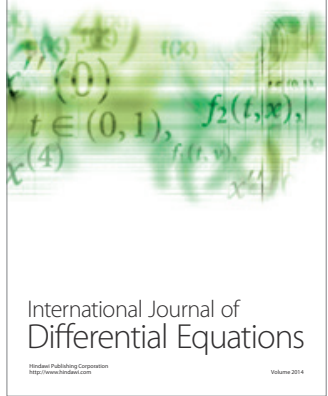
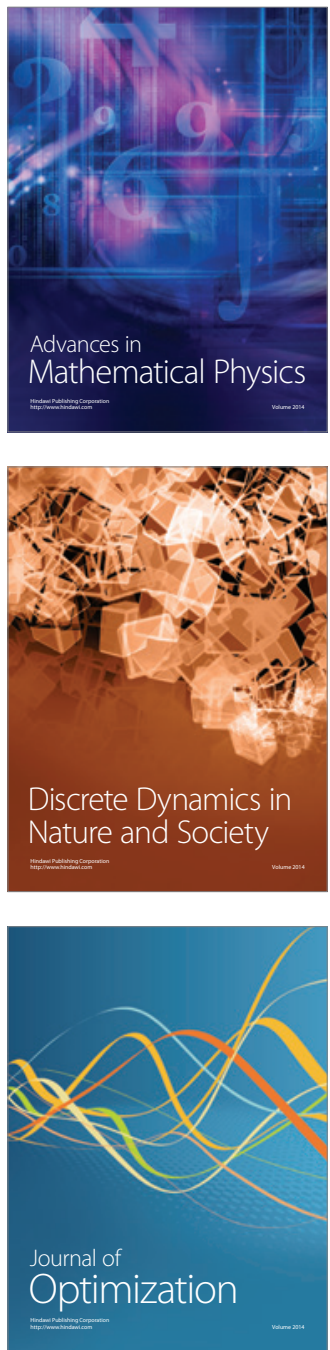